\title{
Albumin-bilirubin grade as a prognostic indicator for patients with non-hepatocellular primary and metastatic liver malignancy undergoing Yttrium-90 radioembolization using resin microspheres
}

\author{
Antoine Azar ${ }^{1}$, Zlatko Devcic ${ }^{2}$, Ricardo Paz-Fumagalli ${ }^{2}$, Lucas Lauar Cortizo Vidal' ${ }^{2}$ J. Mark McKinney ${ }^{2}$, \\ Gregory Frey ${ }^{2}$, Andrew R. Lewis ${ }^{2}$, Charles Ritchie ${ }^{2}$, Jason S. Starr ${ }^{3}$, Kabir Mody ${ }^{3}$, Beau Toskich ${ }^{2}$ \\ ${ }^{1}$ Division of Diagnostic Radiology, ${ }^{2}$ Division of Interventional Radiology, ${ }^{3}$ Division of Oncology, Mayo Clinic Florida, Jacksonville, FL, USA \\ Contributions: (I) Conception and design: A Azar, Z Devcic, R Paz-Fumagalli, B Toskich; (II) Administrative support: None; (III) Provision of study \\ materials or patients: All authors; (IV) Collection and assembly of data: A Azar, LLC Vidal; (V) Data analysis and interpretation: All authors; (VI) \\ Manuscript writing: All authors; (VII) Final approval of manuscript: All authors. \\ Correspondence to: Zlatko Devcic, MD. Division of Interventional Radiology, Mayo Clinic Florida, 4500 San Pablo Rd S, Jacksonville, FL 32224, \\ USA. Email: Devcic.Zlatko@mayo.edu.
}

Background: Studies have shown that the albumin-bilirubin (ALBI) grade can be a superior prognosticator for patients undergoing Yttrium-90 (Y90) glass microsphere radioembolization for hepatocellular carcinoma (HCC) compared to the Child-Pugh (CP) scoring system. Less is known about the applicability of this score in non-hepatocellular malignancies using Y90 resin microspheres. This study evaluates the ALBI grade's ability to predict overall survival and biochemical toxicity in patients undergoing resin Y90 radioembolization and body surface area dosimetry (BSA) for non-hepatocellular primary and metastatic liver malignancies compared to the CP class and Model for End-Stage Liver Disease (MELD) score.

Methods: A retrospective review of patients with intrahepatic metastatic colorectal and neuroendocrine cancers and cholangiocarcinoma undergoing resin radioembolization from 2006-2015 at a single tertiary medical center was performed. ALBI, MELD, and CP scores were compared and correlated with biochemical toxicity and overall survival.

Results: There was a significant difference in overall survival between CP class A and class B liver function $(\mathrm{P}=0.04)$ for the entire patient cohort. ALBI grade $(\mathrm{P}=0.36)$ and MELD score $(\mathrm{P}=0.19)$ were not independently associated with survival. When stratified by CP class, the ALBI grade revealed a trend for survival difference in $\mathrm{CP}$ class $\mathrm{B}(\mathrm{P}=0.05)$. Baseline ALBI grade was associated with post-procedural albumin reduction $(\mathrm{P}=0.01)$ and bilirubin elevation $(\mathrm{P}=0.007)$.

Conclusions: ALBI grade predicted post-procedural biochemical toxicity, but did not predict survival after resin radioembolization of non-hepatocellular liver malignancies using BSA dosimetry. Given the heterogeneity of this study population, dedicated prospective analyses are required.

Keywords: Liver neoplasms; colorectal neoplasms; neuroendocrine carcinoma; cholangiocarcinoma; therapeutic embolization

Submitted Jan 08, 2020. Accepted for publication Mar 02, 2020.

doi: 10.21037/jgo.2020.04.01

View this article at: http://dx.doi.org/10.21037/jgo.2020.04.01 


\section{Introduction}

Radioembolization has a growing role in the management of hepatocellular carcinoma (HCC) (1) as well as non-HCC primary and metastatic liver malignancies (2-4). As such, there is an increased need for prognostic models to identify treatment benefit and recognize risk of toxicity. Assessment of underlying liver function is key in allocation of treatment strategies for patients with HCC (5). The Child-Pugh (CP) $(6,7)$ score has an integral role in the widely-adopted Barcelona Clinic Liver Cancer (BCLC) classification system $(8,9)$ but is limited by the empiric inclusion of the qualitative variables of ascites and encephalopathy, which introduce subjectivity $(10,11)$.

The Model for End Stage Liver Disease (MELD) is based on quantitative variables which are independently predictive of mortality (12) and has been used as an alternative to the $\mathrm{CP}$ score in assessing prognosis in cirrhotic patients (11). However, the MELD score is limited by the lack of universal adoption and objective superiority to the CP score in predicting survival $(13,14)$.

A more recent model devised by Johnson et al., the albumin-bilirubin (ALBI) grade, can stratify survival in patients with cirrhosis, early stage HCC undergoing liver resection, and advanced stage HCC treated with sorafenib (10). The ALBI grade is based on the serum albumin and bilirubin and is therefore not subject to the potential qualitative variable errors present in the $\mathrm{CP}$ score.

While recent studies have found the ALBI grade to be superior to $\mathrm{CP}$ class in discriminating survival in patients with HCC undergoing Yttrium-90 (Y90) radioembolization (15-18), little is known about its applicability for non-HCC primary and metastatic liver malignancies. Furthermore, the ALBI score was studied in patients with predominantly glass microspheres using Medical Internal Radiation Dose (MIRD) methodology (15-18). This study evaluated whether the ALBI grade can predict biochemical toxicity and overall survival in patients with metastatic colorectal cancer (mCRC), intrahepatic cholangiocarcinoma (iCCA), and metastatic neuroendocrine cancers (mNEC) undergoing $\mathrm{Y} 90$ resin microsphere radioembolization using the body surface area dose method (BSA), and compared it to the CP and MELD scoring systems.

\section{Methods}

Institutional review board approval was obtained for this study. A retrospective analysis was performed of all patients undergoing resin microsphere (SIR-Spheres; Sirtex Medical, Lane Cove, Australia) radioembolization of nonHCC liver malignancies from 2006-2015 at a single tertiary medical center. All patients received lobar or staged bilobar radioembolization utilizing BSA dosimetry according to the manufacturer's instructions for use. Patients who received glass microsphere (TheraSphere, Boston Scientific, Marlborough, MA) radioembolizaton, segmental infusions, and ablative or MIRD dosimetry were excluded to reduce the confounding variables of administered activity distribution and mimic the most common utilization of resin microspheres in general practice $(19,20)$. After initial analysis, only patients with intrahepatic mCRC, iCCA, and mNEC were included in order to sufficiently power the study per cohort. Patient demographics were collected at baseline. Laboratory values including albumin, bilirubin, AST, and ALT were collected at baseline and 1-3 and 36 months post-procedure. Baseline CP class was determined prospectively for each patient. Overall survival was defined as time from radioembolization treatment to death. Postprocedural laboratory value changes were graded according to the National Cancer Institute Common Terminology Criteria for Adverse Events v5 (CTCAE) (21) and used to assess toxicity. Toxicity events for all tumor subtypes at 13 months and 3-6 months post-procedure were combined to sufficiently power statistical analysis. CP class, ALBI grade, and MELD score were compared and correlated with survival and laboratory toxicity (CTCAE grade $\geq 2$ ).

\section{Statistical analysis}

An overall comparison between groups for categorical variables was completed using the Chi-square test. Continuous variables were compared using the analysis of variance. Overall survival was estimated using the KaplanMeier method. Comparisons between survival curves between groups were completed using Log-rank tests. Cox proportional hazards models were used to evaluate the relationship of the continuous versions of the variables to the survival endpoint. The relationship of individual variables to the complication outcomes were evaluated using logistic regression models. All analyses were completed using SAS version 9.4 (Cary, NC).

\section{Results}

Ninety-six patients from 2006-2015 met inclusion criteria. Baseline demographics, disease characteristics, and pre- 
Table 1 Patient characteristics

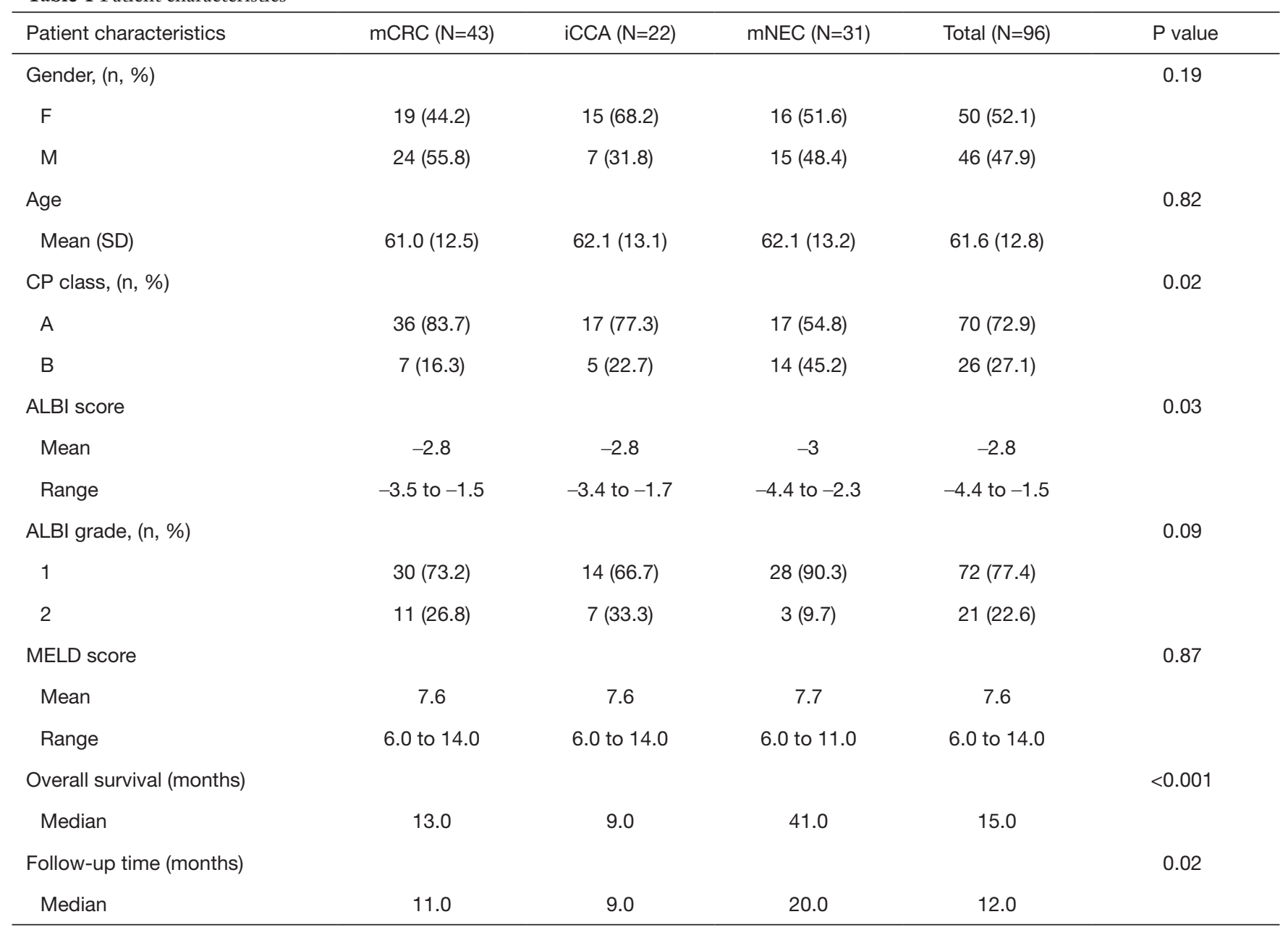

$\mathrm{mCRC}$, metastatic colorectal cancer; iCCA, intrahepatic cholangiocarcinoma; mNEC, metastatic neuroendocrine cancer.

treatment information are listed in Tables 1-3, respectively.

With regards to dosimetry, mean administered activity was $1.5 \mathrm{GBq}$ (range, 0.5-4.7 GBq), without significant difference between the three tumor types $(\mathrm{P}=0.47$, Table 4).

\section{Overall survival}

Among the entire patient population across tumor types there was a significant difference in median survival between patients with CP class A and B (17 vs. 7.5 months, $\mathrm{P}=0.04)$. This significant difference was also demonstrated within the mCRC and iCCA cohorts $(\mathrm{P}<0.001$ and $\mathrm{P}=0.002$ respectively), and there was a trend for survival prediction in mNEC $(\mathrm{P}=0.05)$ (Figure 1). Furthermore, among the entire patient population, sub-stratifying each CP class by ALBI grade 1 and 2 demonstrated no median survival difference for CP A (17 vs. 11 months, $\mathrm{P}=0.69)$, but there was a trend for median survival prediction in CP B (9 vs. 4 months, $\mathrm{P}=0.05$; Figure 2).

There was no statistically significant difference in median survival between ALBI grades 1 and 2 among the whole patient cohort (19 vs. 9 months, $\mathrm{P}=0.36$ ) nor within each of the individual tumor type cohorts ( $\mathrm{mCRC}, \mathrm{P}=0.34$; iCCA, $\mathrm{P}=0.57$; mNEC, $\mathrm{P}=0.07$; Figure 3). When evaluated as a continuous variable the ALBI score was not predictive of survival for the entire group $(\mathrm{P}=0.07)$ or each individual tumor type (mCRC, $\mathrm{P}=0.26$; iCCA, $\mathrm{P}=0.49$; mNEC, $\mathrm{P}=0.33$ ). There was no cutoff value that could predict a survival difference.

Similarly, the MELD score was not associated with survival for all patients $(\mathrm{P}=0.19)$ or when divided into each individual tumor type $(\mathrm{mCRC} \mathrm{P}=0.55$; iCCA $\mathrm{P}=0.14$; 
Table 2 Baseline disease characteristics

\begin{tabular}{|c|c|c|c|c|c|}
\hline Baseline disease characteristics & $\mathrm{mCRC}(\mathrm{N}=43)$ & $\mathrm{iCCA}(\mathrm{N}=22)$ & $\mathrm{mNEC}(\mathrm{N}=31)$ & Total $(\mathrm{N}=96)$ & $P$ value \\
\hline Lobar & 9 (20.9) & $8(36.4)$ & $3(9.7)$ & $20(20.8)$ & \\
\hline Bilobar & $34(79.1)$ & $14(63.6)$ & $28(90.3)$ & $76(79.2)$ & \\
\hline Hepatic tumor burden, (n, \%) & & & & & 0.05 \\
\hline $25-50$ & $8(18.6)$ & $8(36.4)$ & $11(35.5)$ & $27(28.1)$ & \\
\hline $50-75$ & $8(18.6)$ & $6(27.3)$ & $4(12.9)$ & $18(18.8)$ & \\
\hline $75-100$ & $4(9.3)$ & $2(9.1)$ & $8(25.8)$ & $14(14.6)$ & \\
\hline Extrahepatic metastases, (n, \%) & & & & & 0.005 \\
\hline Lymph node metastases, (n, \%) & & & & & 0.6 \\
\hline No & $22(51.2)$ & $14(63.6)$ & $16(51.6)$ & $52(54.2)$ & \\
\hline Yes & $21(48.8)$ & $8(36.4)$ & $15(48.4)$ & $44(45.8)$ & \\
\hline
\end{tabular}

$\mathrm{mCRC}$, metastatic colorectal cancer; iCCA, intrahepatic cholangiocarcinoma; mNEC, metastatic neuroendocrine cancer.

Table 3 Pre-treatment data

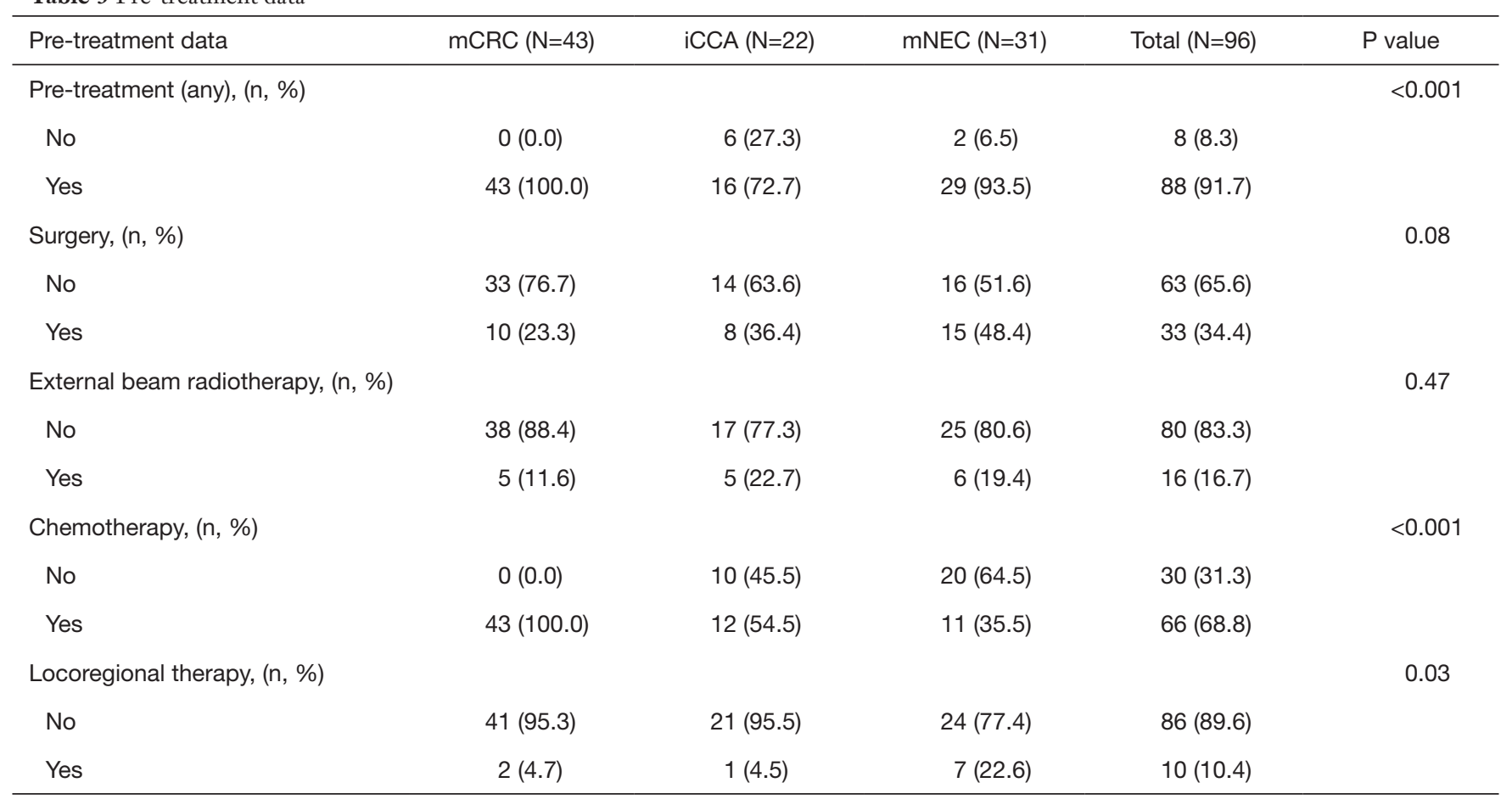

$\mathrm{mCRC}$, metastatic colorectal cancer; iCCA, intrahepatic cholangiocarcinoma; mNEC, metastatic neuroendocrine cancer. 
Table 4 Treatment data

\begin{tabular}{lcccc}
\hline Treatment data & $\mathrm{mCRC}(\mathrm{N}=43)$ & iCCA $(\mathrm{N}=22)$ & $\mathrm{mNEC}(\mathrm{N}=31)$ & Total $(\mathrm{N}=96)$ \\
\hline Administered activity (GBQ) & & & & \\
Mean & 1.4 & 1.5 & 1.7 & 1.5 \\
Range & $0.6-2.5$ & $0.5-2.8$ & $0.7-4.7$ & $0.5-4.7$ \\
\hline
\end{tabular}

$\mathrm{mCRC}$, metastatic colorectal cancer; iCCA, intrahepatic cholangiocarcinoma; mNEC, metastatic neuroendocrine cancer.
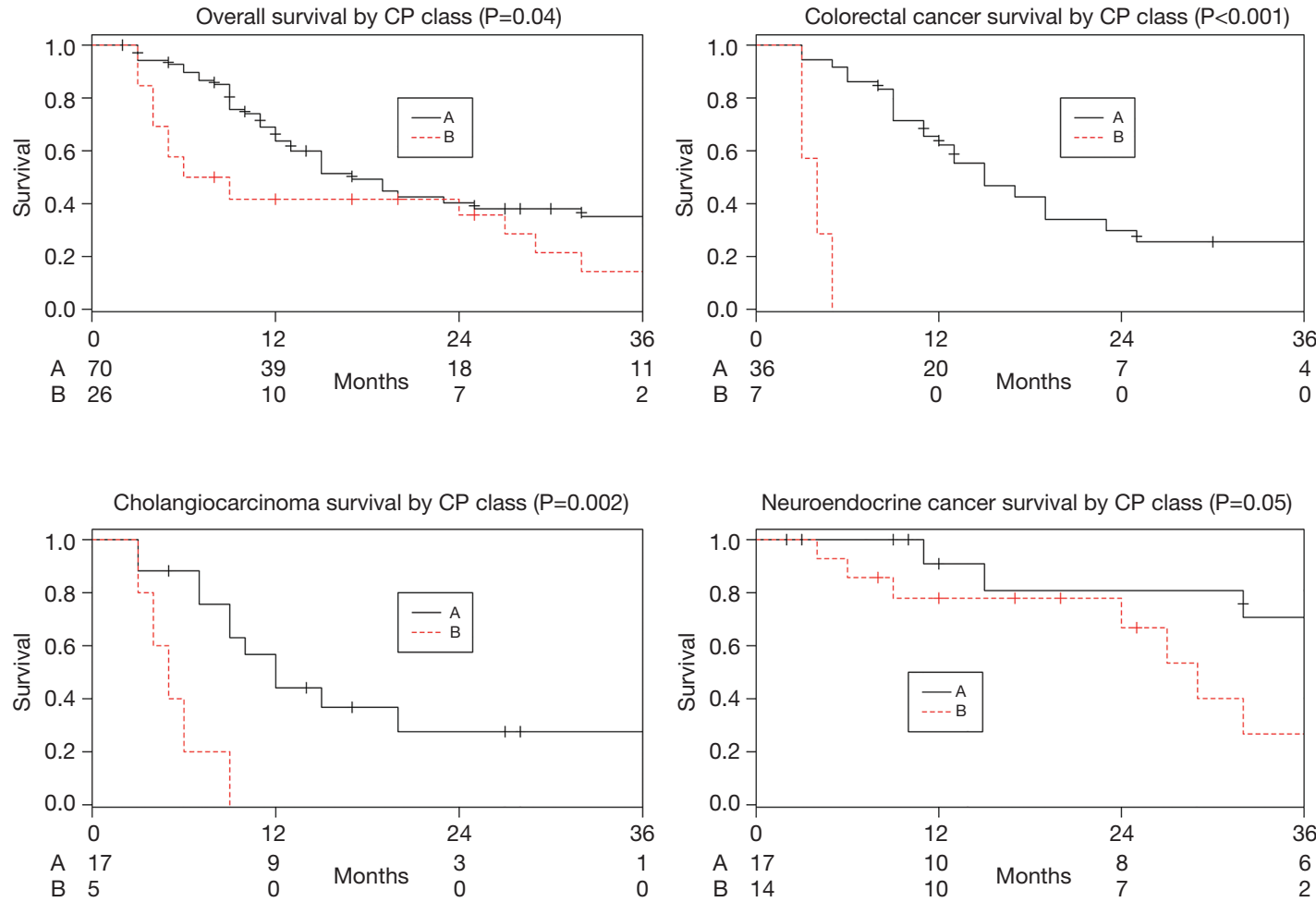

Figure 1 Patient survival by Child-Pugh (CP) Class.
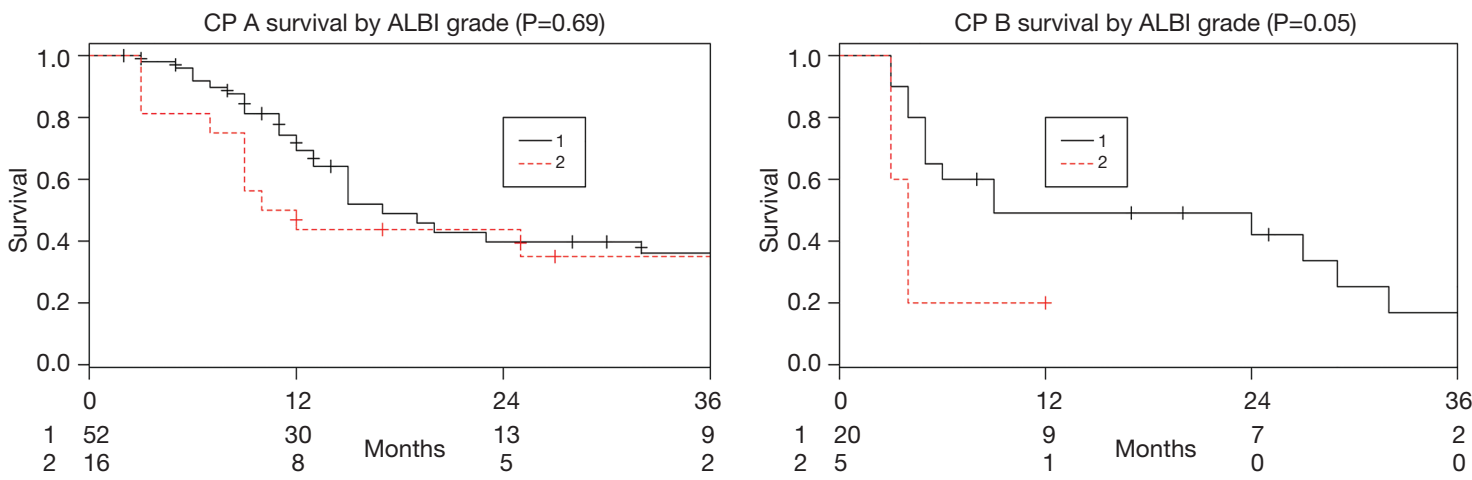

Figure 2 Overall patient survival by Child-Pugh (CP) Class sub-stratified by Albumin-Bilirubin (ALBI) grade. 

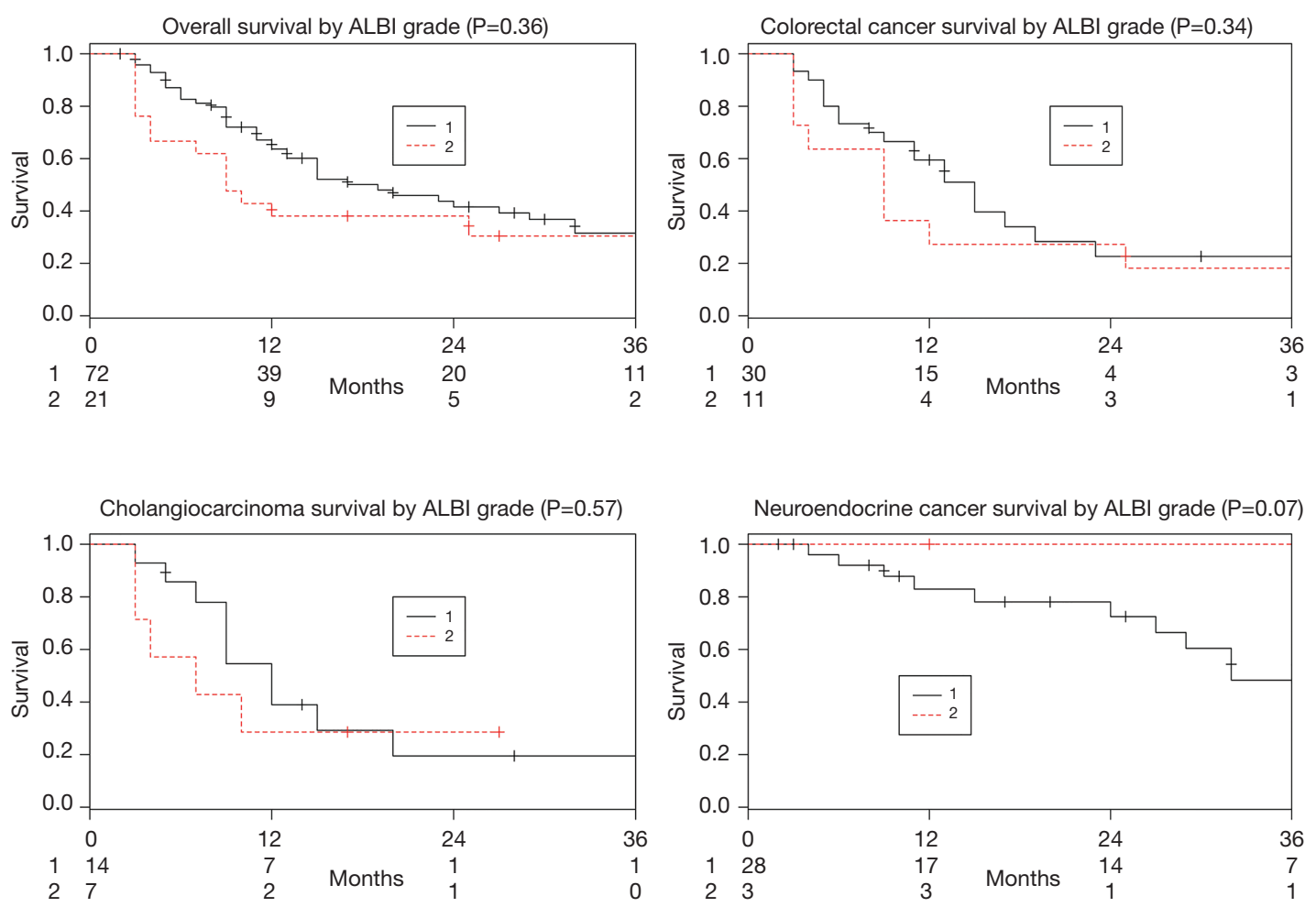

Figure 3 Patient survival by Albumin-Bilirubin (ALBI) grade.

mNEC $\mathrm{P}=0.61$.

\section{Biochemical toxicity}

\section{Albumin}

Patients with ALBI grade 2 were significantly more likely to have a post-procedural decline in their albumin (CTCAE $\geq 2)$ compared to grade $1(\mathrm{P}=0.01)$. There was otherwise no association with $\mathrm{CP}$ class $(\mathrm{P}=0.08)$ or MELD score $(\mathrm{P}=0.32)$.

\section{Bilirubin}

Patients with ALBI grade 2 were significantly more likely to have a post-procedural elevation in their bilirubin (CTCAE $\geq 2)$ compared to grade $1(\mathrm{P}=0.007)$. There was a trend for the $\mathrm{CP} \mathrm{B}$ to predict post-procedural bilirubin elevation, but this did not reach statistical significance $(\mathrm{P}=0.05)$. There was no association with MELD score $(\mathrm{P}=0.48)$.

\section{$A S T$ and $A L T$}

The ALBI grade was not associated with a post-procedural increase in AST (CTCAE $\geq 2)(\mathrm{P}=0.53)$. The MELD score was not associated with a post-procedural increase in AST $(\mathrm{P}=0.99)$ or ALT $(\mathrm{P}=0.12)$. An association could not be determined for CP class and an AST or ALT elevation, or the ALBI grade and an ALT elevation due to the limited number of patients who developed an AST or ALT elevation.

\section{Discussion}

Given the increasing utilization of radioembolization (RE) for hepatocellular (1) and non-hepatocellular primary and metastatic liver malignancies (2-4), there is a need to select patients appropriately and stratify risk of toxicity. The ALBI grade (10) improves prognostication for patients undergoing radioembolization for HCC compared to $\mathrm{CP}(15,16)$ and enables sub-stratification of CP A, BCLC stage A, and BCLC stage $\mathrm{B}$ patients into those with higher probability for survival $(15,16)$. There are no studies to date evaluating the ability of the ALBI grade to predict survival in patients undergoing RE for non-HCC primary and metastatic liver malignancies.

The CP class was able to predict overall survival for 
patients with mCRC and iCCA, and there was a trend for predicting survival in mNEC and for sub-stratifying the CP class B patients into ALBI grades to further refine the survival prediction. The strongest correlation was identified between the CP class and mCRC (Figure 1) which demonstrates a distinct survival advantage to $\mathrm{CP}$ class A. There was no correlation with the ALBI grade or MELD score, and there was no cut-off value in the ALBI score which predicted survival. While there was a trend between ALBI grade 1 and worse survival outcomes for patients with mNEC, this is likely due to the small number of mNEC patients who had baseline ALBI grade 2 in our cohort (3 patients), which limits statistical analysis. Although the ALBI grade has a clear value in assessing survival in patients with HCC (10), it may not be superior to the CP score in predicting outcomes in patients with non-HCC malignancies.

The CP score may be more predictive in these patient populations as it identifies the presence of ascites, a factor which may represent more severe disease involvement in patients without underlying cirrhosis (22). The CP score may also be capturing the effects of hepatotoxic systemic therapy exposure which is frequent in patients with nonHCC malignancies (23). Finally, the presence of liver dysfunction limits the choices of systemic therapy available to patients with metastatic liver malignancy (24), and information on how to best approach these patients remains an active area of study (25). This may expose patients to additional toxicity from altered pharmacokinetics of antineoplastic agents in the presence of any physiologic dysfunction that results in the development of ascites (26), which is better captured by the CP score than by ALBI.

Compared to MELD and ALBI, the CP class provided improved prognostication, especially for patients with $\mathrm{mCRC}$, and may be helpful in treatment algorithms weighing the risks, benefits, expected results and time to benefit of various therapies.

Post-procedural biochemical toxicity was the secondary endpoint of this study. Gui et al. had found no association between ALBI grade and post-procedural adverse events (including elevation in bilirubin) in patients undergoing radioembolization for HCC (16). While other studies have evaluated CP scores and biochemical toxicity in HCC patients (27), none have evaluated the ability of the ALBI grade, CP class, or MELD score to predict biochemical toxicity in patients undergoing radioembolization for nonhepatocelluar cancers involving the liver. In a combined cohort of patients with all three tumor subtypes, post- procedural reductions in albumin and elevations in bilirubin were the only biochemical toxicities associated with the ALBI grade. The CP class and MELD score had no associations. Predicting biochemical toxicity prior to RE can facilitate the development of treatment strategies, as liver dysfunction may limit the ability to offer future systemic therapy $(23,25)$. In patients with baseline liver dysfunction, it provides value in making treatment decisions and identifying those at increased risk for hepatotoxicity requiring closer monitoring and clinical intervention.

\section{Limitations of this study}

This study was limited by its retrospective nature as well as the heterogeneity of tumor types included in the analysis and their underlying biology. The degree of differentiation of disease in the neuroendocrine cohort was not controlled for. The results were not controlled for the volume of extrahepatic disease burden; although this is somewhat mitigated by the institutional practice to offer locoregional therapy only to patients with liver dominant disease. Certain analyses were limited by small patient numbers, such as in some assessments of biochemical toxicity. Most patients were on highly variable chemotherapy regimens over extended periods of time which were also not controlled for, but common in palliative radioembolization clinical practice. Tumor mutational profiling to control for disease biology was not available for the entirety of the study cohort. Finally, the radioembolization lobar volumetrics were not available for analysis, however is unlikely to affect the results of the study as there was no significant difference in lobar disease distribution among the tumor subtypes $(\mathrm{P}=0.06)$ and bilateral treatments are common with resin microspheres in patients with metastatic disease. In recognition of these limitations, this study aimed to generate an initial perspective on the role for the ALBI score in a commonly heterogeneous population receiving Y90 resin microsphere radioembolization.

\section{Conclusions}

The ALBI grade predicts post procedural biochemical toxicity but not survival in pateints with non-hepatocellular liver malignancy treated with resin microsphere radioembolization using BSA dosimetry. Further study is required to determine the validity of this conclusion with dedicated, disease specific, studies. 


\section{Acknowledgments}

We would like to acknowledge Dr. Weiping Wang and Dr. David Livingston for their assistance with data collection. Funding: None.

\section{Footnote}

Conflicts of Interest: All authors have completed the ICMJE uniform disclosure form (available at http://dx.doi. org/10.21037/jgo.2020.04.01). BT is an advisor to Boston Scientific and Johnson and Johnson. KM reports personal fees from Astra Zeneca, personal fees from Bayer, personal fees from Celgene, personal fees from Eisai, personal fees from Exelixis, personal fees from Ipsen, grants from Agios, grants from ArQule, grants from Senwha Biosciences, grants from Taiho, grants from Genetech Incyte, grants from Medimmune, grants from Puma Biotechnology. The other authors have no conflicts of interest to declare.

Ethical Statement: The authors are accountable for all aspects of the work in ensuring that questions related to the accuracy or integrity of any part of the work are appropriately investigated and resolved. The research was conducted in accordance with the Declaration of Helsinki (as revised in 2013). The study was approved by the Institutional Review Board of Mayo Clinic Florida. Individual consent for this retrospective analysis was waived.

Open Access Statement: This is an Open Access article distributed in accordance with the Creative Commons Attribution-NonCommercial-NoDerivs 4.0 International License (CC BY-NC-ND 4.0), which permits the noncommercial replication and distribution of the article with the strict proviso that no changes or edits are made and the original work is properly cited (including links to both the formal publication through the relevant DOI and the license). See: https://creativecommons.org/licenses/by-nc-nd/4.0/.

\section{References}

1. Tohme S, Bou Samra P, Kaltenmeier C, et al. Radioembolization for Hepatocellular Carcinoma: A Nationwide 10-Year Experience. J Vasc Interv Radiol 2018;29:912-9.e2.

2. Raval M, Bande D, Pillai AK, et al. Yttrium-90 radioembolization of hepatic metastases from colorectal cancer. Front Oncol 2014;4:120.
3. Mouli S, Memon K, Baker T, et al. Yttrium-90 radioembolization for intrahepatic cholangiocarcinoma: safety, response, and survival analysis. J Vasc Interv Radiol 2013;24:1227-34.

4. Jia Z, Wang W. Yttrium-90 radioembolization for unresectable metastatic neuroendocrine liver tumor: A systematic review. Eur J Radiol 2018;100:23-9.

5. Bruix J, Sherman M. Management of hepatocellular carcinoma: An update. Hepatology 2011;53:1020-2.

6. Child CG, Turcotte JG. Surgery and portal hypertension. Major Probl Clin Surg 1964;1:1-85.

7. Pugh RN, Murray-Lyon IM, Dawson JL, et al. Transection of the oesophagus for bleeding oesophageal varices. $\mathrm{Br} \mathrm{J}$ Surg 1973;60:646-9.

8. Kinoshita A, Onoda H, Fushiya N, et al. Staging systems for hepatocellular carcinoma: Current status and future perspectives. World J Hepatol 2015;7:406-24.

9. Llovet JM, Bru C, Bruix J. Prognosis of hepatocellular carcinoma: the BCLC staging classification. Semin Liver Dis 1999;19:329-38.

10. Johnson PJ, Berhane S, Kagebayashi C, et al. Assessment of liver function in patients with hepatocellular carcinoma: a new evidence-based approach-the ALBI grade. J Clin Oncol 2015;33:550-8.

11. Durand F, Valla D. Assessment of prognosis of cirrhosis. Semin Liver Dis 2008;28:110-22.

12. Malinchoc M, Kamath PS, Gordon FD, et al. A model to predict poor survival in patients undergoing transjugular intrahepatic portosystemic shunts. Hepatology 2000;31:864-71.

13. Durand F, Valla D. Assessment of the prognosis of cirrhosis: Child-Pugh versus MELD. Journal of Hepatology 2005;42:S100-7.

14. Peng Y, Qi X, Guo X. Child-Pugh Versus MELD Score for the Assessment of Prognosis in Liver Cirrhosis: A Systematic Review and Meta-Analysis of Observational Studies. Medicine (Baltimore) 2016;95:e2877.

15. Hickey R, Mouli S, Kulik L, et al. Independent Analysis of Albumin-Bilirubin Grade in a 765-Patient Cohort Treated with Transarterial Locoregional Therapy for Hepatocellular Carcinoma. J Vasc Interv Radiol 2016;27:795-802.

16. Gui B, Weiner AA, Nosher J, et al. Assessment of the Albumin-Bilirubin (ALBI) Grade as a Prognostic Indicator for Hepatocellular Carcinoma Patients Treated With Radioembolization. Am J Clin Oncol 2018;41:861-6.

17. Antkowiak M, Gabr A, Das A, et al. Prognostic Role of Albumin, Bilirubin, and ALBI Scores: Analysis of 1000 
Patients with Hepatocellular Carcinoma Undergoing Radioembolization. Cancers 2019;11:879.

18. Mohammadi H, Abuodeh $\mathrm{Y}$, Jin W, et al. Using the Albumin-Bilirubin (ALBI) grade as a prognostic marker for radioembolization of hepatocellular carcinoma. J Gastrointest Oncol 2018;9:840-6.

19. van Hazel GA, Heinemann V, Sharma NK, et al. SIRFLOX: Randomized Phase III Trial Comparing FirstLine mFOLFOX6 (Plus or Minus Bevacizumab) Versus mFOLFOX6 (Plus or Minus Bevacizumab) Plus Selective Internal Radiation Therapy in Patients With Metastatic Colorectal Cancer. J Clin Oncol 2016;34:1723-31.

20. Wasan HS, Gibbs P, Sharma NK, et al. First-line selective internal radiotherapy plus chemotherapy versus chemotherapy alone in patients with liver metastases from colorectal cancer (FOXFIRE, SIRFLOX, and FOXFIRE-Global): a combined analysis of three multicentre, randomised, phase 3 trials. Lancet Oncol 2017;18:1159-71.

21. US National Cancer Institute. Common Terminology Criteria for Adverse Events (CTCAE) Version 5.0. National Institutes of Health. 2017. Available online: https://ctep.cancer.gov/protocolDevelopment/

Cite this article as: Azar A, Devcic Z, Paz-Fumagalli R, Vidal LLC, McKinney JM, Frey G, Lewis AR, Ritchie C, Starr JS, Mody K, Toskich B. Albumin-bilirubin grade as a prognostic indicator for patients with non-hepatocellular primary and metastatic liver malignancy undergoing Yttrium-90 radioembolization using resin microspheres. J Gastrointest Oncol 2020;11(4):715-723. doi: 10.21037/jgo.2020.04.01
electronic_applications/docs/CTCAE_v5_Quick_ Reference_8.5x11.pdf

22. Sangisetty SL, Miner TJ. Malignant ascites: A review of prognostic factors, pathophysiology and therapeutic measures. World J Gastrointest Surg 2012;4:87-95.

23. Field KM, Dow C, Michael M. Part I: Liver function in oncology: biochemistry and beyond. Lancet Oncol 2008;9:1092-101.

24. Pinter M, Trauner M, Peck-Radosavljevic M, et al. Cancer and liver cirrhosis: implications on prognosis and management. ESMO Open 2016;1:e000042.

25. Cabibbo G, Palmeri L, Palmeri S, et al. Should cirrhosis change our attitude towards treating non-hepatic cancer? Liver Int 2012;32:21-7.

26. Li J, Gwilt P. The effect of malignant effusions on methotrexate disposition. Cancer Chemother Pharmacol 2002;50:373-82.

27. Abouchaleh N, Gabr A, Ali R, et al. (90)Y Radioembolization for Locally Advanced Hepatocellular Carcinoma with Portal Vein Thrombosis: LongTerm Outcomes in a 185-Patient Cohort. J Nucl Med 2018;59:1042-8. 